\title{
Programming and Activating Cyberparks: An Introduction and Overview
}

\author{
Michiel de Lange $\mathrm{e}^{1(\mathbb{\varpi})}$ (1) and Martijn de Waal $^{2}$ (]) \\ ${ }^{1}$ Utrecht University, Utrecht, The Netherlands \\ m. 1.delange@uu.nl \\ 2 Amsterdam University of Applied Sciences, Amsterdam, The Netherlands \\ b.g.m.de.waal@hva.nl
}

\section{Introduction: Programming and Activating Cyberparks}

Part 3 Programming and Activating Cyberparks deals with the variety of ways in which urban public spaces can be reinvigorated through the use of digital media technologies. As is outlined in the introduction to this volume, digital media technologies profoundly shape the use and perception of urban public spaces. Critical observers have noted that digital media may threaten the public nature of our cities and civic spaces. For instance, elsewhere we have described these threats in terms of three Cs: commercialisation, control, and capsularisation (de Lange and de Waal 2013). First, the combination of digital media technologies and consumer culture overlays everyday urban life with a market logic of pervasive customer tracing, quantification, and a vying for attention. Datafication and personalized recommendation services capitalise on our habitual everyday movements in the city, turning them into an ever-expanding string of (potential) customer 'touchpoints'. This affects the spatial, social and cultural dimensions of almost every realm of urban life, from work to meeting to leisure to travel to home. Visible illustrations include the rapidly changing appearance of high streets in most cities, or the nature and quality of inner-city neighbourhoods coinciding with the popularity of platforms like Airbnb (for more on platforms, see van Dijck et al. 2018). As a result, our polyvocal and frictional public open spaces are being transformed into silent and seamless marketplaces, where public interactions are reduced to commercial transactions.

Second, cities are being equipped with a range of technologies pervasive control. Governments in the post-9/11 landscape have sought ways to monitor, react and increasingly often also pre-emptively act to calamities in order to secure urban life (Crang and Graham 2007; Crandall 2010). New technologies of control include crime maps, CCTV surveillance, urban dashboards based on real-time data, and smart algorithms and AI that can detect out-of-the-ordinary signals (e.g. loud impact noises) and behaviour (e.g. outdoor group gatherings). Crang and Graham (2007) paint a bleak scenario in which these technologies have turned our cities into quasi-militarised zones aimed at maximising transparency where nothing out of the ordinary is allowed.

Third, interactions in public open spaces are increasingly characterised by the widespread availability of personal and portable media technologies like mobile 
phones, tablets, laptops, portable audio devices, smart watches, and so on, plus ubiquitous access to networked content (e.g. Netflix, Spotify), and networked social relations through social media. This drives a tendency towards retreating in one's personal media bubble, which some have described as capsularisation (De Cauter 2004) or cocooning (Ito et al. 2009).

How can we wrest public open spaces from these developments? The chapters in part 3 suggest a range of possible approaches to this challenge. Some of the key words in this part include playfulness, hackability, the commons, resilience, placemaking, civic participation, local knowledge and cultural heritage. The series of chapters taken together form a variegated and incomplete mosaic of possible entry points to engage with this question. They are not so much answers as much as suggested venues for further exploration and experimentation. In a sense, what connects these interventions is an underlying drive towards making the city 'hackable', that is, open to interventions and systemic forms of city-making by non-experts (de Lange and de Waal 2019).

\section{Overview of Chapters}

The chapter Smart Citizens in the Hackable City: On the Datafication, Playfulness, and Making of Urban Public Spaces through Digital Art (3.2) by Michiel de Lange, Kåre Synnes, Gerald Leindecker explores a variety of people-centric narratives as corollaries to the dominance of technologically driven smart city visions. They argue that smart city visions can be criticized for lacking a perspective on the inclusion of citizens in city-making, for emphasizing efficiency at the expense of urban public life, and for its technology-centeredness in which technological fixes for complex societal issues are suggested. To counterbalance this dominant narrative, the authors forward a range of alternative options. People-centric strategies include playfulness, collaborative forms of datafication and visualization, the use of interactive (media) art and public installations, and new types of making and hacking in the city. While none of these narratives provide the same attractive promise of solving urban woes through technology, they do have the advantage of placing people at the forefront rather than an afterthought. They emphasize the capacity for change that city inhabitants have.

In their chapter Using ICT in the Management of Public Open Space as a Commons (3.3) Georgios Artopoulos, Paschalis Arvanitidis and Sari Suomalainen argue that public open spaces (POS) are not just simply physical spaces, but should be understood as a commons, or common pool resource. Narratives play an important role in the formation of public open spaces as assemblages. These narratives include cultural codings of a place, and the practices, users and uses that are understood as belonging to particular places. In contemporary cities, ICT use becomes part of these assemblages. Particular technologies and services may open up the construction of narratives around places or provide new means through which POS can be managed. This means that process of appropriation of public open spaces can become more open, and pluralistic. And in turn, this could contribute to a process in which, as the authors write, stakeholders "collectively develop institutional arrangements [..], which enable them to ensure proper use and longevity of the common pool resource." In the development of that argument, the chapter contributes to the discussion of Public Open Spaces by showing how new mobile ICT could help communities to collectively 
appropriate POS and bring out an understanding of these public open spaces as common pool resources that can be managed by local communities.

In their contribution Revealing the Potential of Public Places: Adding a New Digital Layer to The Existing Thematic Gardens in Thessaloniki Waterfront (3.4) Tatiana Ruchinskaya, Konstantinos Ioannidis and Kinga Kimic argue for the inclusion of ICT in place making strategies to activate public spaces. They base their study on an analysis of the thematic gardens developed at the Thessaloniki waterfront. These open urban public spaces were to revive the gardens existing in Thessaloniki in the 19th and 20th century that had been demolished in the urban expansions of the 1960s and 1970s. The new gardens were laid out as a string of fifteen sites at the seafront, expressing the relationship between the city and the sea. Each garden has its own theme. Walking through them can be experienced as a spatial narrative in which various symbolic meanings unfold. An analysis and survey of the site found that visitors highly appreciate this thematization. However, many users note that the gardens were often empty and lacked a number of facilities to make them truly attractive. While the original design didn't include any digital amenities, the authors lay out a range of options for the application of site-specific digital layers that could enhance the experience and usefulness of the Gardens. They see potential in combining the themed design of the gardens with digital narrative experiences and adding digital platforms that could be used to share knowledge or personal experiences. Digital media thus might be used to add new symbolic layers, turning the gardens in an example of hybrid read-write publishing surfaces. Hence, the chapter gives an overview of various strategies for the hybridization of Public Open spaces that could be beneficial for designers and policy makers.

Konstantinos Lalenis, Balkiz Yapicioglou and Petja Ivanova-Radovanova, in their chapter Cyberpark, a New Medium of Human Associations, a Component of Urban Resilience (3.5), analyse how the resilience of public open spaces can be increased by integrating cyberparks into its spatial planning and policies. Urban resilience is understood in two ways. First, as the capacity to absorb a sudden crisis or disaster in the short run, and second as the capacity of cities to integrate this robustness into infrastructures and governance in the long run. The authors underline that 'resilient thinking' has become an increasingly imported concept in urban planning to update important physical and societal systems for future adaptations. Cyberparks, as digitalphysical hybrids of public open spaces, are significant resources in urban resilience, as they combine physical infrastructure and digitally mediated information that may aid in disaster management. In a case study of the refugee crisis is Greece, the chapter shows how this works in practice.

In the contribution A Spotlight of Co-Creation and Inclusiveness of Public Open Spaces (3.6), Ina Šuklje Erjavec and Tatiana Ruchinskaya focus on co-creation to engage a variety of stakeholders with everyday urban environments based on equality, diversity and social cohesion. Different from public participation and citizen engagement, co-creation as a method entails a special type of collaboration, an act of collective creativity where people are working or acting together with others to create something that is not known in advance. The authors then proceed to connect this notion of co-creation to the 'Four-D Model', which highlights four types of civic engagement: discover, debate, decide and do. The authors analyse the potential strengths of digital media technologies for fostering these four aspects of civic engagement with public open spaces in co-creation processes. The chapter also gives an overview of a large number of digital tools available for these purposes. 
The last contribution to part 3 is the chapter CyberParks Songs and Stories Enriching Public Spaces with Localized Culture Heritage Material such as Digitized Songs and Stories (3.7) by Kåre Synnes, Georgios Artopoulos, Carlos Smaniotto Costa, Marluci Menezes, and Gaia Redaelli. The chapter looks at the ways in which technologies can contribute to archiving and accessing intangible cultural heritage. It does so by examining a concept for a heritage app called CyberParks Songs and Stories. This app aims to increase the understanding of European cultures through localized storytelling. By analysing three cases that are listed as Intangible Cultural Heritage by UNESCOs Intergovernmental Committee for the Safeguarding of the Intangible Cultural Heritage, the authors highlight how users can contribute their own narratives and insights to the platform. In doing so, the concept addresses the next big challenge in cultural heritage after the digitisation phase, which is how to manage and opening up the vast troves of data in meaningful ways and involve people in contributing content.

\section{References}

Crandall, J.: The geospatialization of calculative operations: tracking, sensing and megacities. Theor. Cult. Soc. 27(6), 68-90 (2010)

Crang, M., Graham, S.: Sentient cities: ambient intelligence and the politics of urban space. Inf. Commun. Soc. 10(6), 789-817 (2007)

De Cauter, L.: The Capsular Civilization: On the City in the Age of Fear. NAi Publishers, Rotterdam (2004)

Dijck, J., Poell, T., Waal, M.: The Platform Society. Oxford University Press, New York (2018)

Ito, M., Okabe, D., Anderson, K.: Portable objects in three global cities: the personalization of urban places. In: Ling, R.S., Campbell, S.W. (eds.) The Reconstruction of Space and Time: Mobile Communication Practices, pp. 67-87. Transaction Publishers, New Brunswick. (2009)

de Lange, M., de Waal, M.: Owning the city: new media and citizen engagement in urban design. First Monday, 18(11) (2013)

de Lange, M., de Waal, M. (eds.): The Hackable City: Digital Media and Collaborative Citymaking in the Network Society. Springer, London (2019). https://doi.org/10.1007/978-98113-2694-3

Open Access This chapter is licensed under the terms of the Creative Commons Attribution 4.0 International License (http://creativecommons.org/licenses/by/4.0/), which permits use, sharing, adaptation, distribution and reproduction in any medium or format, as long as you give appropriate credit to the original author(s) and the source, provide a link to the Creative Commons license and indicate if changes were made.

The images or other third party material in this chapter are included in the chapter's Creative Commons license, unless indicated otherwise in a credit line to the material. If material is not included in the chapter's Creative Commons license and your intended use is not permitted by statutory regulation or exceeds the permitted use, you will need to obtain permission directly from the copyright holder.

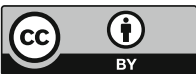

